\title{
Comparison between platelet-rich plasma injections and hyaluronic acid injections in the treatment of patellar tendinopathies: a randomized trial.
}

\author{
J.-F. Kaux ${ }^{1,2}$, S. Bornheim¹, N. Dardenne ${ }^{3}$, R. Deroisy ${ }^{2}$, A. Samson ${ }^{1,2}$, \\ M. Roberjot ${ }^{1}$, J.-L. Croisier ${ }^{1,2}$ \\ 1 Department of Sports and Rehabilitation Sciences, University of Liège, Belgium \\ 2 Department of Physical Medicine and Sports Traumatology, SportS2, FIFA Medical Centrer of Excellence, \\ University and University Hospital of Liège, Belgium \\ 3 Biostatistic department, University of Liège, Belgium
}

\author{
CORRESPONDING AUTHOR: \\ Jean-François kaux \\ Physical Medicine and Sport \\ Traumatology, \\ University Hospital of Liège, \\ Avenue de l'Hôpital, B35, \\ 4000 Liège, Belgium, \\ E-mail: jfkaux@chu.ulg.ac.be, \\ Tel: +32 43668241 \\ DOI: \\ 10.32098/mltj.03.2019.03
}

LEVEL OF EVIDENCE: 2

\begin{abstract}
SUMMARY
Background. As patellar tendinopathies are frequently chronic there has been plenty of research carried out to develop effective treatments, among which platelet-rich plasma (PRP) and hyaluronic acid (HA).

Hypothesis. Therefore we aimed to compare the effect after three months following injections of PRP compared with HA injections on patients with a proximal patellar tendinopathy. Methods. 33 sportsmen with chronic proximal patellar tendinopathies were included and randomised into 2 groups and treated respectively with PRP (obtained using an apheresis machine) or HA injections. To assess the evolution of the pathology, algo-functional tests (VISA-P and IKDC) and isokinetic tests were carried out pre-injection, after 6 weeks and after 3 months post-injections.

Results. The results of VAS, algometric scores, IKDC scores and VISA-P showed a considerable improvement in the two groups over time $(\mathrm{p}<0.01)$, except for the US findings. Isokinetic tests showed significant modifications for the quadriceps in $\mathrm{C} 60(\mathrm{p}=0.01)$ for the pathological limb only at the end of the follow-up period. VAS associated with isokinetic tests decreased significantly for all contraction modes after three months in the HA group $(\mathrm{p}<0.05)$. Correlations showed that there was a significant negative association between VAS and strength in C60, C240 and E30 ( $<<0.01)$ only for the PRP group.

Conclusion. Both PRP and HA can alleviate the symptoms of proximal patellar tendinopathy in the medium term. However, only PRP leads to a decrease in pain associated with an increase in the strength of the quadriceps.
\end{abstract}

\section{KEY WORDS}

patellar tendon; tendinopathy; jumper's knee; isokinetic; strength

\section{INTRODUCTION}

Patellar tendinopathies are particularly frequent in various sports, especially where athletes perform many jumps/landings as in volleyball, basketball or the triple jump, etc. (1) Its treatment can be difficult and the condition often remains chronic (2). This is the reason why new treatments have been developed, among which feature platelet-rich plasma (PRP) injections (3). Some clinical tests have previously evaluated the effect of one or multiple PRP injections in the treatment of proximal patellar tendinopathies (4-7). Even if there exists a lack of general agreement concerning its preparation and use, and that recent systematic reviews are controversial, some of these have concluded that PRP could be appropriate as a treatment in such indications (8-12).

Recently, the anti-inflammatory and viscoelastic properties of hyaluronic acid (HA) on liquid connective tissue have been suggested for the treatment of tendinopathies (13). Some fundamental studies show encouraging results concerning HA's 
ability to promote tendon gliding and reduce adhesion as well as to improve the tendon's architectural organisation (14-16). Some observations also support its use in a clinical environment to diminish pain and improve function $(17,18)$. Moreover, some tests with patients suffering from various tendinopathies have demonstrated that HA injections can lead to better clinical results than with corticosteroid injections (19-21).

We aimed to compare the effect of PRP versus HA injections after three months on patients who have a proximal patellar tendinopathy.

\section{METHODS}

All experimental procedures and protocols used in this investigation were reviewed and approved by the Ethics Committee of our University. Our protocol (PRP collection and injection, rehabilitation post-injection, assessments during the follow-up) has been described previously (22-24). Our study was registered as clinical trial 2010-021957-38 (amended) by the "Agence Fédérale des Médicaments et des Produits de Santé" (AFMPS) in Belgium. The authors confirm that all ongoing and related trials for this intervention are registered.

A power calculation was carried out to determine the ideal number of subjects required to observe a statistically significant difference in the VAS scale compared with previous studies. This showed that, with 10 subjects per group, a power of $80 \%$ at the $5 \%$ critical level, and a standard deviation of 2.5 if such a difference exists, would be suitable.

Thirty-three patients (all male), with chronic (more than 3 months) proximal patellar tendinopathy, diagnosed clinically and confirmed by ultrasound scans (US) and/or magnetic resonance imaging (MRI), without bone conflict or bone edema, were enrolled and randomized into 2 groups (PRP or HA) from 1 October 2015 to 30 June 2017 (Figure 1). One criterion for inclusion was for patients to have had an unresponsive experience with other classical conservative treatments (painkillers, NSAIDs or physiotherapy, including eccentric rehabilitation and shock wave therapy). They were fully randomised into two groups (PRP and HA respectively), having first signed an informed consent form. Complete assessments were made for each subject using the same timing and a variety of scores: 10-Point Visual Analog Scale (VAS); Clinical examination using a pressure Algometer Commander (JTech Medical Industries, Salt Lake City, Utah); International Knee Documentation Committee form (IKDC); Victorian Institute of Sport Assessment-Patellar questionnaire (VISA-P); Isokinetic assessment, performed using a Cybex Norm (Cybex International, Medway, Massachusetts, USA) after a 10-min warm-up on a cycle ergometer. The modalities evaluated were concentric $60^{\circ} \mathrm{s}^{-1}(\mathrm{C} 60)$ and $240^{\circ} \mathrm{s}^{-1}$ (C240), and eccentric $30^{\circ} \mathrm{s}^{-1}$ (E30) on both quadriceps. A VAS score was used to quantify pain resulting from each test; US (Logiq S7, GE Healthcare, Chicago, Illinois). A complete evaluation was made before the infiltrations, and then repeated by the same examiner at an interval of 6 weeks and 3 months after the last injection.

Before platelets were collected, a haematological cell count for each patient was performed under laboratory conditions. PRP was obtained using an apheresis machine (COM. TEC and kit CS5L, Fresenius-Kabi, Bad-Homburg, Germany) (25). We decided to collect platelets with a concentration of around $8.5 \times 10^{5}$ platelets/microL (almost no leukocytes or erythrocytes). Immediately prior to infiltration, 300 microL of $\mathrm{CaCl}_{2}$ were added to the PRP to activate the platelets. Six milliliters of PRP were injected once only into the patellar tendon of subjects of group 1 after disinfection. Only one perforation in the hypo-echoic area of the tendon was carried out under US guidance (24). Local anaesthetic was not used in order to avoid a decrease in platelet activity linked to a $\mathrm{pH}$ modification (26).

The subjects in group 2 received two peri-tendinous HA $2 \%(40 \mathrm{mg} / 2 \mathrm{~mL}$, Ostenil Tendon, TRB Chemedica International SA, Geneva, Switzerland) injections of $2 \mathrm{~mL}$ under US guidance at one week apart $(19,21)$. No local anaesthetic was used as for group 1.

Patients in both groups were given local cryotherapy immediately following the infiltration. In case of pain, they were forbidden any anti-inflammatory drugs, but allowed to use type I or II classical pain killers.

After 5 to 7 days of relative rest, patients commenced a standardised progressive sub-maximal eccentric program, consisting of 5 sessions of 15 repetitions 3 times a week and supervised by a physiotherapist (27). This eccentric exercise was carried out using the weight of the patient's body, with the back against the wall. The patient would slowly slide his back down the wall until his pathologic leg bent to a $60^{\circ}$ angle before pushing on both legs to return to the starting position. A thirty second rest period was allowed between sessions. The patient was also treated with electrostimulation, stretching of the quadriceps, and cryotherapy. After one week of rehabilitation, a second infiltration of 2 $\mathrm{mL}$ of HA was administered to the subjects in Group 2, following the same protocol. Five to seven days after the second infiltration, the sub-maximal eccentric protocol was again commenced for another 5 week period (supervised by a physiotherapist). The angulation and the number of sessions were both progressively increased during the rehabilitation, from $60^{\circ}$ to $90^{\circ}$ and from 5 to 7 sessions of 15 to 20 repetitions, respectively. Ten minutes of cycloergometer exercise, using low resistance, was also added to the program 2 weeks after the second treatment, and proprio- 
ception exercises were introduced after 1 month. No sporting activities were authorised before 6 weeks after the injection. After this period, a progressive and sports-specific training was restarted under medical supervision with the aim of returning completely to the sport after 3 months.

Results were expressed as the mean \pm standard deviation of the mean (SD). Normality was investigated by comparing the mean and median, by plotting histogram and QQ-plot. The Shapiro-Wilk test was also performed. At time 0, the two groups were compared using a Student's t-test for independent samples. Evolution over time for both groups was studied using generalised linear mixed models (GLMM). The association between quantitative parameters was tested using the Pearson's (r) or Spearman's correlation (rs) coefficient or asymmetric distributions. A coefficient $>0$ indicates an increasing relationship between variables, a coefficient $<0$ a decreasing relationship. Correlations less than 0.3 were considered as negligible, between 0.3 and 0.5 as low, between 0.5 and 0.7 as moderate and greater than 0.7 as high. Results were considered significant at a level of uncertainty of $5 \%(p<0.05)$. Statistical analyses were conducted using Statistical Analysis System version 9.4 software (SAS Institute, Cary, North Carolina).

\section{RESULTS}

Eighteen subjects (group 1: aged 29.5 \pm 9.9 years; $78.9 \pm 12.8 \mathrm{~kg}$; $177.4 \pm 6.9 \mathrm{~cm})$ received a 6-milliliter intra-tendinous leukocyte-poor PRP injection (obtained using an aphaeresis machine) (25) under US guidance. The other fifteen subjects (group 2: aged $29.3 \pm 8.1$ years; $77.1 \pm 10.6 \mathrm{~kg} ; 178.1 \pm 5.8 \mathrm{~cm}$ ) received two peri-tendinous $\mathrm{HA} 2 \%(40 \mathrm{mg} / 2 \mathrm{~mL})$ injections $(2 \mathrm{~mL})$ under US guidance one week apart.

At baseline, no significant differences were observed between the 2 groups except for algometer results in favour of the PRP group ( $\mathrm{p}=0.019$ ).

Fourteen out of 18 subjects $(77,8 \%)$ in the PRP group and 11 out of 15 subjects $(73,3 \%)$ in the HA group were responders to the treatments (decrease of pain $>50 \%$ ).

The results of VAS $(\mathrm{p}<0.01)$, algometric scores $(\mathrm{p}<0.01)$, IKDC scores $(\mathrm{p}<0.01)$ and VISA-P $(\mathrm{p}<0.01)$ showed a considerable improvement in the two groups with time (Figure 1), except for the US findings. For group 1 (Table I), isokinetic tests showed significant modifications for the quadriceps with an improvement of maximum peak torque in $\mathrm{C} 60(\mathrm{p}=0.01)$ for the pathological limb at the end of follow-up. For group 2, the improvement of quadriceps maximum peak torque at $\mathrm{C} 240^{\circ} / \mathrm{s}$ was significant $(\mathrm{p}<0.01)$ for the pathological knee only 6 weeks post injections. In this group, VAS associated with isokinetic tests decreased significantly for all contraction modes after three months of the study.

Correlations showed that there was a significant negative association between VAS and strength in C60 ( $\mathrm{r}=-0.66, \mathrm{p}=$ $0.0030)$, C240 ( $\mathrm{r}=-0.56, \mathrm{p}=0.016)$, and E30 ( $\mathrm{rs}=-0.66, \mathrm{p}=$ 0.0030 ) only for the PRP group but these associations were moderate There was no significant association between the VAS score and strength for the HA group $(\mathrm{p}>0.05)$.

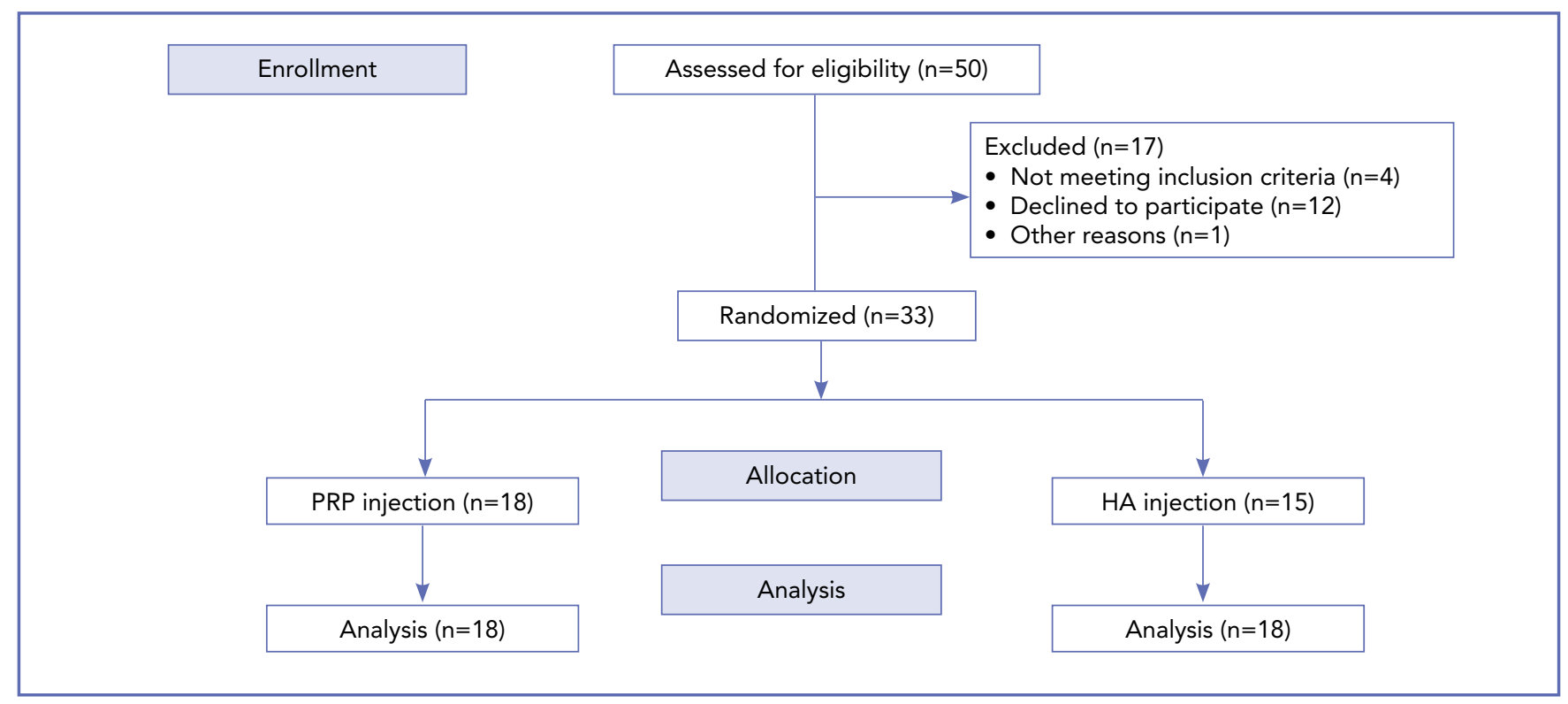

Figure 1: CONSORT flowchart. 
Table I. Results for the isokinetic assessment for both groups at the 3 monitoring points. Quad C60 or C240: Quadriceps concentric $60 \%$ s or $240 \%$; Quad E30: Quadriceps eccentric $30 \% \mathrm{~s}^{*} ; \mathrm{p}<0.05$ between T0 and T2**; $p<0.05$ between T0 and T3***; $p<0.05$ between T2 and T3. T0: before injection; T1: 6 weeks after injection; T2: 3 months after injection.

\begin{tabular}{llll}
\hline Group 1 & T0 & T1 & T2 \\
\hline Quad C60 $($ N.m) & $167.11 \pm 49.12$ & $($ mean \pm SD) & (mean \pm SD) \\
\hline VAS C60 & $2.63 \pm 2.92$ & $167.27 \pm 48.96$ & $159.38 \pm 46.42^{* * *}$ \\
\hline Quad C240 $($ N.m) & $114.33 \pm 30.91$ & $1.69 \pm 1.87$ & $2.00 \pm 1.79$ \\
\hline VAS C240 & $2.00 \pm 2.77$ & $114.38 \pm 34.98$ & $115.94 \pm 33.12$ \\
\hline Quad E30 (N.m) & $170.94 \pm 88.17$ & $1.44 \pm 2.02$ & $1.11 \pm 1.03$ \\
\hline VAS E30 & $4.66 \pm 3.47$ & $191.83 \pm 76.07$ & $192.66 \pm 77.45$ \\
\hline Group 2 & T0 & $2.97 \pm 2.49$ & $3.33 \pm 2.42$ \\
\hline Quad C60 (N.m) & (mean \pm SD) & T1 & T2 \\
\hline VAS C60 & $163.33 \pm 39.95$ & (mean \pm SD) & (mean \pm SD) \\
\hline Quad C240 (N.m) & $3.93 \pm 2.57$ & $157.06 \pm 35.27$ & $161.40 \pm 36.72$ \\
\hline VAS C240 & $98.33 \pm 21.26$ & $2.90 \pm 2.46$ & $2.33 \pm 2.49^{* *}$ \\
\hline Quad E30 (N.m) & $3.53 \pm 2.35$ & $108.73 \pm 20.70^{*}$ & $107.66 \pm 21.46$ \\
\hline VAS E30 & $190.66 \pm 44.81$ & $1.33 \pm 1.58^{* *}$ & $1.06 \pm 1.48^{* *}$ \\
\hline
\end{tabular}
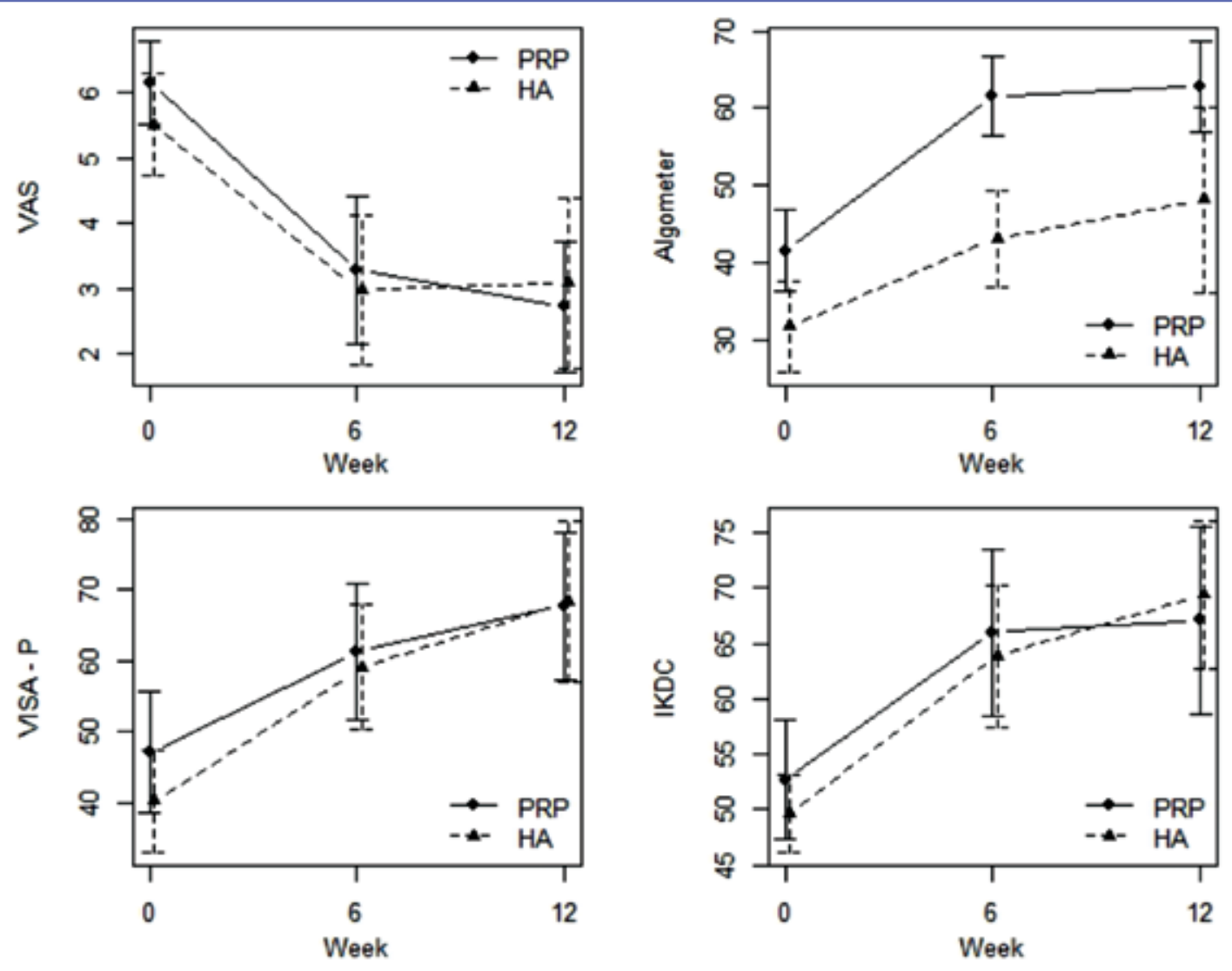

Figure 2. Results for the VAS, algometer and algo-functional scores (VISA-P and IKDC) for both groups at the 3 monitoring points. All the graphs show a significant improvement over time $(p<0.01)$. 


\section{DISCUSSION}

While some patellar tendinopathies evolve favourably with traditional conservative treatments (NSAIDs, physiotherapy/rehabilitation, shock wave therapy, etc.), others remain chronic. It is for this reason that a lot of research has been carried out to develop or adapt treatments for such rebel conditions, among which PRP and HA $(13,28)$.

Some clinical studies have previously evaluated the effect of PRP in the treatment of proximal patellar tendinopathies (6, 23, 24, 29). Even if this treatment remains controversial in the literature, recent systematic reviews have concluded that PRP could be recommend (low evidences) as a treatment in such indications (8-12). The use of HA in the treatment of tendinopathies is even more recent in clinical practice, even if pre-clinical studies in the healing process of tendons have been reported in the past 20 years $(13,14,30)$. However, it seems that HA injections have been effective in small groups of patients with tendinopathies (13).

The PRP used in this study was a leukocyte-poor PRP with a concentration of 850,000 platelets/microliter which is usually recommended in other studies $(26,31)$. To collect platelets, an apheresis machine was used to obtain the same platelet concentration for each patient (25). Moreover, only one intra-tendinous injection was carried out as we demonstrated that it was not necessary to replicate them (24). HA is classically used in cases of osteoarthritis (32). Recently, it has been adapted to be used in the treatment of tendinopathies (13). In these cases, HA is concentrated to $2 \%$ and also contains mannitol which confers anti-inflammatory properties $(13,33)$. Two injections were carried out as in previous studies and also as recommended in these papers (19, $21,30)$. Hyaluronic acid could have the ability to promote tendon gliding and reduce adhesion as well as to improve the architectural organisation (13).

As PRP needs to be associated with rehabilitation to be effective, we added it to our post-injection protocol even though it is not an obligation after HA injection. We are reminded that all patients had no success with conservative management techniques, including physiotherapy, prior to inclusion.

In our study, which is the first to compare intra-tendinous injections of PRP with those of HA, both under US guidance, we wanted to compare the effects of both treatments on chronic patellar tendinopathies with a follow-up after 3 months to evaluate the medium term effects. We observed that both PRP and HA can alleviate the symptoms, especially pain, of such conditions. As for isokinetic assessment, a significant improvement in quadriceps strength was observed after an injection of PRP while no improvement was observed after 2 injections of HA. However, we observed that pain decreases during the assessment for the 3 contraction modes in the HA group. In view of the standard deviations of VAS during isokinetic assessment at the end of follow-up, even if there is a significant overall improvement, some patients remain in considerable pain even in the HA group. HA seems to have a quicker pain-killing effect during functional assessments than PRP, which is known to be effective only after a minimum of 3 months, and that this improvement continues for up to 1 year $(8$, 13). Thus, a longer follow-up should be used to evaluate the long term evolution of these two treatments, but both treatments can be considered in cases of patellar tendinopathies for intermediate terms ( 3 months), even if there are more proof points in the literature for PRP injections. However, the correlations showed that the increasing strength in C60, C240 and E30 is related to a decrease in pain evaluated with VAS only with the PRP group. This observation is really essential in the rehabilitation of patellar tendinopathy which can lead to a loss of quadriceps strength linked to a decrease in their use.

Our study presents some limitations: a) we compared 2 groups with infiltrations of PRP and HA, but we did not have a real control group. However, the aim was to evaluate outcomes of 1 and 2 infiltrations of PRP, not to compare the effect of PRP against a control group; b) as we compared 1 infiltration of PRP to 2 infiltrations 15 days apart, evaluations of Group 2 took place 2 weeks after those of Group 1. However, the evaluation delay after the last infiltration was identical for both groups - 6 weeks and 3 months.

\section{CONCLUSIONS}

Both PRP and HA can alleviate the symptoms of proximal patellar tendinopathy in the medium term. HA can be considered as a new treatment for tendinopathies. However, only PRP leads to a decrease in pain associated with an increase in the strength during isokinetic assessment. Studies with a more long term follow-up should be carried out.

\section{Conflicts of interest}

TRB Chemedica provided the HA injections (Ostenil Tendon) free of charge for all the subjects in this study.

\section{REFERENCES}

1. Kaux, J.F., et al., Current opinions on tendinopathy. J Sports Sci Med, 2011. 10(2): pp. 238-53.

2. Kaux, J.F. and J.M. Crielaard, [Tendon et tendinopathie]. Journal de Traumatologie du Sport, 2014. 31(4): pp. 235-240. 
3. Kaux, J.F., et al., Tendinopathies and platelet-rich plasma (PRP): from pre-clinical experiments to therapeutic use. J Stem Cells Regen Med, 2015. 11(1): pp. 7-17.

4. Rowan, T.L. and J.L. Drouin, A multidisciplinary approach including the use of platelet-rich plasma to treat an elite athlete with patellar tendinopathy - a case report. J Can Chiropr Assoc, 2013. 57(4): pp. 301-9.

5. Zayni, R., et al., Platelet-rich plasma as a treatment for chronic patellar tendinopathy: comparison of a single versus two consecutive injections. Muscles Ligaments Tendons J, 2015. 5(2): pp. 92-8.

6. Charousset, C., et al., Are multiple platelet-rich plasma injections useful for treatment of chronic patellar tendinopathy in athletes? a prospective study. Am J Sports Med, 2014. 42(4): pp. 906-11.

7. Dragoo, J.L., et al., Platelet-rich plasma as a treatment for patellar tendinopathy: a double-blind, randomized controlled trial. Am J Sports Med, 2014. 42(3): pp. 610-8.

8. Andia, I., et al., Platelet-rich plasma in the conservative treatment of painful tendinopathy: a systematic review and meta-analysis of controlled studies. Br Med Bull, 2014. 110(1): pp. 99-115.

9. Balasubramaniam, U., R. Dissanayake, and L. Annabell, Efficacy of platelet-rich plasma injections in pain associated with chronic tendinopathy: a systematic review. Phys Sportsmed, 2015: pp. 1-9.

10. Dupley, L. and C.P. Charalambous, Platelet-Rich Plasma Injections as a Treatment for Refractory Patellar Tendinosis: A Meta-Analysis of Randomised Trials. Knee Surg Relat Res, 2017. 29(3): pp. 165-171.

11. Di Matteo, B., et al., Platelet-rich plasma: evidence for the treatment of patellar and Achilles tendinopathy-a systematic review. Musculoskelet Surg, 2015. 99(1): pp. 1-9.

12. Fitzpatrick, J., M. Bulsara, and M.H. Zheng, The Effectiveness of Platelet-Rich Plasma in the Treatment of Tendinopathy: A Meta-analysis of Randomized Controlled Clinical Trials. Am J Sports Med, 2017. 45(1): pp. 226-233.

13. Kaux, J.F., A. Samson, and J.M. Crielaard, Hyaluronic acid and tendon lesions. Muscles Ligaments Tendons J, 2015. 5(4): pp. 264-9.

14. Salti, N.I., R.J. Tuel, and D.P. Mass, Effect of hyaluronic acid on rabbit profundus flexor tendon healing in vitro. J Surg Res, 1993. 55(4): pp. 411-5.

15. Nishida, J., et al., Effect of hyaluronic acid on the excursion resistance of tendon grafts. A biomechanical study in a canine model in vitro. J Bone Joint Surg Br, 2004. 86(6): pp. 918-24.

16. Oryan, A., et al., Repeated administration of exogenous Sodium-hyaluronate improved tendon healing in an in vivo transection model. J Tissue Viability, 2012. 21(3): pp. 88-102.

17. Merolla, G., P. Bianchi, and G. Porcellini, Ultrasound-guided subacromial injections of sodium hyaluronate for the management of rotator cuff tendinopathy: a prospective comparative study with rehabilitation therapy. Musculoskelet Surg, 2013. 97 Suppl 1: pp. 49-56.

18. Kumai, T., et al., The short-term effect after a single injection of high-molecular-weight hyaluronic acid in patients with enthesopathies (lateral epicondylitis, patellar tendinopathy, insertional Achilles tendinopathy, and plantar fasciitis): a preliminary study. J Orthop Sci, 2014. 19(4): pp. 603-11.
19. Lynen, N., Treatment of chronic tendinopathies with peritendinous hyaluronan injections under sonographic guidance an interventional, prospective, single-arm, multi center study. Orthopädische und Unfallchirurgische Praxis, 2012. 1(10): pp. 7-11.

20. Gorelick, L., et al., Lateral epicondylitis injection therapy: a safety and efficacy analysis of hyaluronate versus corticosteroid injections. Adv Tech Biol Med, 2015. 3(2): pp. 1000130.

21. Lynen, N., et al., Comparison of Peritendinous Hyaluronan Injections Versus Extracorporeal Shock Wave Therapy in the Treatment of Painful Achilles' Tendinopathy: A Randomized Clinical Efficacy and Safety Study. Arch Phys Med Rehabil, 2017. 98(1): pp. 64-71.

22. Kaux, J.F., et al., One injection of platelet-rich plasma associated to a submaximal eccentric protocol to treat chronic jumper's knee. J Sports Med Phys Fitness, 2015. 55(9): pp. 953-61.

23. Kaux, J.-F, et al., One-year follow-up of platelet-rich plasma infiltration to treat chronic proximal patellar tendinopathies. Acta Orthop Belg, 2015. 81(2):251-6.

24. Kaux, J.F., et al., Using platelet-rich plasma to treat jumper's knees: Exploring the effect of a second closely-timed infiltration. J Sci Med Sport, 2016. 19(3):200-204.

25. Moog, R., et al., Evaluation of a concurrent multicomponent collection system for the collection and storage of WBC-reduced RBC apheresis concentrates. Transfusion, 2001. 41(9): pp. 1159-64.

26. Kaux, J.F., et al., Reflections about the optimisation of the treatment of tendinopathies with PRP. Muscles Ligaments Tendons J, 2015. 5(1): pp. 1-4.

27. Kaux, J.F., et al., Description of a standardized rehabilitation program based on sub-maximal eccentric following a platelet-rich plasma infiltration for jumper's knee. Muscles Ligaments Tendons J, 2014. 4(1): pp. 85-9.

28. Kaux, J.F. and J.M. Crielaard, [Tendinopathies et plasma riche en plaquettes (PRP) : Applications cliniques. Revue de la littérature]. Journal de Traumatologie du Sport, 2012. 29(3): pp. 174-178.

29. Andia, I. and N. Maffulli, Use of platelet-rich plasma for patellar tendon and medial collateral ligament injuries: best current clinical practice. J Knee Surg, 2015. 28(1): pp. 11-8.

30. Fogli, M., N. Giordan, and G. Mazzoni, Efficacy and safety of hyaluronic acid $(500-730 \mathrm{kDa})$ Ultrasound-guided injections on painful tendinopathies: a prospective, open label, clinical study. Muscles Ligaments Tendons J, 2017. 7(2): pp. 388-395.

31. Kaux, J.F. and T. Emonds-Alt, The use of platelet-rich plasma to treat chronic tendinopathies: A technical analysis. Platelets, 2018. 29(3):213-227.

32. Bruyère, O., et al., A consensus statement on the European Society for Clinical and Economic Aspects of Osteoporosis and Osteoarthritis (ESCEO) algorithm for the management of knee osteoarthritis-From evidence-based medicine to the real-life setting. Semin Arthritis Rheum, 2016. 45(4 Suppl): pp. S3-11.

33. Abate, M., C. Schiavone, and V. Salini, The use of hyaluronic acid after tendon surgery and in tendinopathies. Biomed Res Int, 2014. 2014: pp. 783632. 